\title{
Stage III Nasopharyngeal Carcinoma AJCC v6
}

National Cancer Institute

\section{Source}

National Cancer Institute. Stage III Nasopharyngeal Carcinoma A/CC v6. NCI Thesaurus.

Code C7396.

Stage III includes: (T1, N2, M0); (T2a, N2, M0); (T2b, N2, M0); (T3, N0, M0); (T3, N1, M0); (T3, N2, M0). T3: T umor involves bony structures and/or paranasal sinuses. N2:

Bilateral metastasis in lymph node(s), $6 \mathrm{~cm}$ or less in greatest dimension, above the supraclavicular fossa. M0: No distant metastasis. (AJCC 6th ed.) 